\title{
Enfermedades cardiovasculares y cáncer: ¿dos entidades mutuamente relacionadas?
}

\author{
Evelyn Mendoza-Torres ${ }^{1,2}$, Roberto Bravo-Sagua ${ }^{3}$, Mónica Villa ${ }^{1}$, Nancy Flores $^{1}$, María José Olivares ${ }^{1}$, Ximena Calle, Jaime A Riquelme ${ }^{1}$, \\ Claudia Bambs ${ }^{4}$, Pablo Castro ${ }^{5}$, Sergio Lavandero ${ }^{1,6}$ \\ 1. Advanced Center for Chronic Diseases (ACCDiS), Facultad de Ciencias Químicas y Farmacéuticas y Facultad de Medicina, Universidad de \\ Chile, Santiago, Chile. \\ 2. Facultad de Ciencias de la Salud, Universidad Libre Seccional Barranquilla, Colombia. \\ 3. Advanced Center for Chronic Diseases (ACCDiS), Instituto de Nutrición y de los Alimentos (INTA), Universidad de Chile, Santiago, Chile. \\ 4. Advanced Center for Chronic Diseases (ACCDiS), Departamento de Salud Pública, Facultad de Medicina, Pontificia Universidad Católica de \\ Chile, Santiago, Chile. \\ 5. Advanced Center for Chronic Diseases (ACCDiS), División de Enfermedades Cardiovasculares, Facultad de Medicina, Pontificia Universidad \\ Católica de Chile, Santiago, Chile. \\ 6. Departamento de Medicina Interna (División Cardiología), University of Texas Southwestern Medical Center, Dallas, Texas, USA.
}

Las enfermedades cardiovasculares y el cáncer son enfermedades crónicas transmisibles culturalmente, y las dos causas principales de mortalidad en el mundo. Además del gran impacto sobre la mortalidad y morbilidad, estas enfermedades han mostrado un alto grado de relación entre ellas debido, entre otras razones, a que comparten factores de riesgo y mecanismos biológicos. La alta incidencia de enfermedad cardiovascular en pacientes con cáncer es un fenómeno conocido que ha orientado el desarrollo del campo interdisciplinario de la cardio-oncología. Sin embargo, en la última década han surgido evidencias que muestran el papel que desempeñan las enfermedades cardiovasculares en el desarrollo de cáncer. Un estudio reciente publicado por Meijers y cols, en agosto de 2018 en Circulation, mostró que la insuficiencia cardiaca post-infarto del miocardio contribuye significativamente al desarrollo del cáncer de colón, apoyando lo obtenido en estudios epidemiológicos anteriores. Este estudio también sugiere que el crecimiento tumoral podría producirse por factores secretados por el corazón insuficiente abriendo un amplio grupo de posibilidades de investigación en lo que sería un nuevo campo de la medicina cuyo propósito sería el desarrollo de nuevas estrategias para el seguimiento y tratamiento del cáncer en pacientes con enfermedades cardiovasculares. El presente artículo revisa los factores de riesgo, y mecanismos celulares y moleculares, que son comunes en las enfermedades cardiovasculares y el cáncer, la contribución del trabajo de Meijers y cols hacia un mayor entendimiento de la interrelación entre estas patologías y las perspectivas futuras con respecto a los nuevos hallazgos.

Palabras clave: enfermedades cardiovasculares, cáncer, insuficiencia cardiaca, factores de riesgo. 


\section{Cardiovascular Diseases and cancer: ¿mutually related entities?}

Cardiovascular diseases and cancer are culturally transmitted chronic diseases and the two main causes of death globally. In addition to their high morbidity and mortality, these diseases are closely related, due to their common risk factors and biological mechanisms. The high incidence of cardiovascular diseases in cancer patients is widely known phenomenon, which has oriented the development of the interdisciplinary field of cardio-oncology Nonetheless, there is emerging evidence in the last decade suggesting a potential role for cardiovascular diseases in the onset of cancer. A recent publication by Meijers et al in the scientific cardiovascular journal Circulation showed that heart failure significantly contributes to tumor growth, confirming previous epidemiological findings suggesting this hypothesis. Moreover, this study indicates that tumor growth may be stimulated by the secretion of factors from the failing heart, opening a wide spectrum of research areas in what may be suggested as a new field in medicine that would seek to develop new strategies to treat and prevent cancer in patients with cardiovascular diseases. This article will review shared risk factor and common cellular and molecular pathways in cardiovascular diseases and cancer, the contribution of Meijers et al to a better understanding of the connection of these diseases and future perspectives in light of the new evidence. Key words: cardiovascular diseases, cancer, heart failure, risk factors. 
Enfermedades crónicas trasmisibles culturalmente El concepto original de enfermedades crónicas no transmisibles incluye el cáncer, diabetes, enfermedad pulmonar obstructiva crónica, obesidad y enfermedades cardiovasculares. Estas patologías se caracterizan por ser de larga duración, progresión lenta, incurables y resultan de la combinación de factores genéticos, fisiológicos, ambientales y conductuales tales como la industrialización, pobreza, tabaquismo, alcohol, alimentos procesados e inactividad física ${ }^{1-4}$. El término no transmisible y las características citadas anteriormente solo entregan una visión muy parcial de estas enfermedades. No abordan sus condiciones de transmisibilidad social y cultural, generando importantes limitaciones debido a que el problema es observado solo desde el comportamiento individual y no desde el entorno social, proporcionando muy poca relevancia a los antecedentes sociogenéticos.

A partir de la propuesta de Allen \& Feigl (2017) ${ }^{1}$, se está debatiendo el cambio del nombre de las enfermedades crónicas no transmisibles, por uno que permita apreciar la transmisión social y cultural de estas enfermedades. Para ello se debe analizar de manera global sus factores de riesgo, los cuales poseen una naturaleza antropogénica y socialmente contagiosa, siendo influenciados por las condiciones económicas, intergeneracionales y culturales, como muestra el estudio de Christakis \& Fowler (2007), donde se describe la trasmisión de la obesidad a través de los vínculos sociales, determinando la importancia de las relaciones interpersonales y no de las distancias geográficas en la inducción y difusión de la obesidad $^{5}$.

El término "no transmisible" genera un menor impacto por considerarse "no problemático", derivando en una percepción de menor importancia que, a su vez, conlleva a una baja inversión en los recursos destinados a prevención y estudio de las enfermedades crónicas no transmisibles en comparación con las enfermedades infecciosas. Esta percepción influye desde los gobiernos, pasando por la sociedad hasta el sector privado. A partir de las evidencias mencionadas, se fundamenta la búsqueda de un nuevo concepto de las enfermedades crónicas no transmisibles que se ajuste a su condición de transmisión social y cultural. Al debatir sobre esta nomenclatura, se espera influir positivamente a las acciones preventivas y al estudio de las mismas, permitiendo fomentar la innovación, acción multisectorial, aumento de recursos y oportunidad para asegurar un enfoque más equilibrado e integrado para establecer las prioridades de salud pública.

Estas enfermedades son la principal causa de muerte en el mundo (responsables del $80 \%$ del total de muertes) ${ }^{6}$. En Chile, cerca del $84 \%$ de la población fallece debido a ellas, significando un gran costo económico para el país y para las personas que las poseen. La última Encuesta Nacional de Salud 2016-17 (ENS) corrobora la alta prevalencia de factores de riesgo y baja de factores protectores $^{7,8}$ (Figura 1), además, permite visualizar una sociedad con desigualdades también a nivel de las enfermedades de acuerdo con la situación educacional, edad y sexo. A través de estos resultados se observan que los esfuerzos son insuficientes y no logran controlar la incidencia de estas enfermedades en nuestra sociedad ${ }^{8-10}$.

Impacto de las dos principales enfermedades crónicas transmisibles culturalmente: enfermedades cardiovasculares y el cáncer.

Entre las enfermedades crónicas, las cardiovasculares y el cáncer son las principales causas de muerte a nivel mundial. Las enfermedades cardiovasculares lideran las estadísticas, causando 17,7 millones de muertes por año, seguidas por el cáncer, responsable de 8.8 millones de muertes ${ }^{6}$. En Chile, las enfermedades cardiovasculares y el cáncer generan más de la mitad de las muertes por año, correspondientes a $27,1 \%$ y $25,8 \%$, respectivamente $^{11}$. La idea de un potencial vínculo entre ambas enfermedades no es nueva y, actualmente, estas enfermedades ya no son consideradas entidades independientes, debido al creciente número de evidencias que sugieren una estrecha relación entre ellas ${ }^{12}$.

\section{Factores de riesgo comunes entre las enfermedades} cardiovasculares y el cáncer

El cáncer y las enfermedades cardiovasculares comparten factores de riesgo y mecanismos biológicos que pueden dar cuenta de su interconexión y, a la vez, confundir o enmascarar una posible relación causa-efecto ${ }^{13}$. Entre los factores de riesgo se encuentran el tabaquismo, sedentarismo, dislipidemia, diabetes mellitus, hipertensión $\operatorname{arterial~y~obesidad~}^{12,13}$. Dentro del mecanismo que comparten estas patologías cabe destacar la inflamación crónica, el estrés oxidativo y la autofagia ${ }^{14-23}$.

La inflamación crónica es una respuesta inmune persistente, inicialmente local y a largo plazo sistémica, que está presente en estos dos grupos de enfermedades y que es clave, tanto en su patogénesis, como en su progresión 14,22,23. Este proceso, en ambos casos, genera la activación de células del sistema inmune, las 


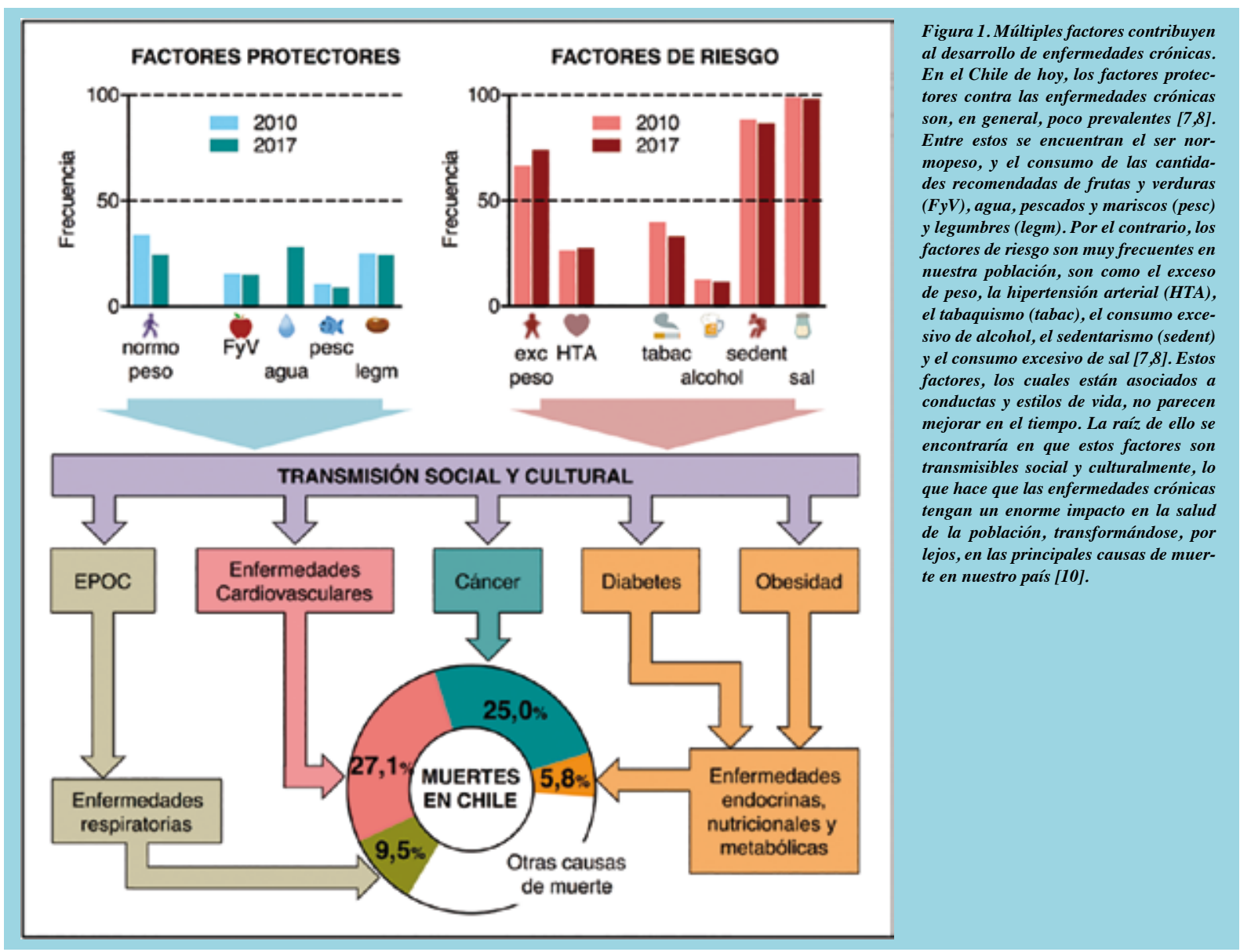

cuales activan algunas vías de señales (NF $x$ B, HIF-1, STAT) que promueven la expresión de los principales mediadores inflamatorios (TNF- $\alpha$, IL-1, IL-6, TGF- $\beta 1$, COX-2, iNOS), activación de fibroblastos y células endoteliales, aumento de proteínas de adhesión (VCAM1, ICAM-1, p-selectinas), síntesis de factores de crecimiento (PDGF y FGF) e incremento en la producción de especies reactivas del oxígeno (ROS) ${ }^{13,25-27}$. Existe evidencia que varias de estas moléculas participan directamente en la patogenia de estas enfermedades y se encuentran elevadas en la sangre de pacientes con estas patologías $^{28-31}$. Por ejemplo, TGF- $\beta 1$, PDGF, FGF y VCAM-1 son claves en el proceso de aterosclerosis y se ha descrito que aumentan tanto la proliferación de células tumorales como la angiogénesis ${ }^{28,32-40}$. A su vez el incremento de las ROS lleva al desarrollo de estrés oxidativo, el que también contribuye activamente a la progresión de estas patologías, el caso del cáncer produce daño de DNA, activación de proto-oncogenes y aumento del microambiente inflamatorio ${ }^{41}$. En el caso de ECV, estas especies contribuyen a la oxidación del
LDL que es punto de inicio de la formación de la placa de ateroma y en isquemia/reperfusión produce daño cardíaco $^{42,43}$. El tercer mecanismo común entre ECV y cáncer es la autofagia, esta vía media la degradación de proteínas y de organelos dañados, lo que permite reciclar componentes celulares en condiciones de carencia de nutrientes o frente a condiciones de estrés, como el estrés oxidativo ${ }^{44}$. Cuando la homeostasis no logra ser recuperada, este proceso culmina con la muerte celular ${ }^{45}$. Existen antecedentes de que la activación de la autofagia favorece el desarrollo de diferentes tipos de cáncer $^{17-19}$. En el caso de las ECV, se ha descrito que tanto el aumento, como su disminución, contribuyen a la patogenia de estas enfermedades 20,21 . A su vez, ha sido descrito que varias citoquinas producidas en procesos inflamatorio pueden regular la autofagia ${ }^{46}$.

\section{Cardio-oncología}

La incidencia de la enfermedad cardiovascular como consecuencia indirecta del cáncer ha sido extensamente estudiada. Desde hace varios años se ha descrito que 
los pacientes que sobreviven al cáncer tienen una mayor mortalidad a largo plazo por causa de las enfermedades cardiovasculares, debido a que la mayoría de las terapias contra el cáncer, tanto la radioterapia como la quimioterapia, son potencialmente tóxicas para el corazón $\mathrm{n}^{47,48}$. En el contexto de lo indicado, las antraciclinas son fármacos ampliamente descritos como cardiotóxicos. El mecanismo por cual ejercen su efecto contempla la generación de ROS, así como la unión a topoisomerasa tipo II para así generar disfunción mitocondrial ${ }^{49}$. Sin embargo, los medicamentos anti-cancerígenos tienen un alto espectro de cardiotoxicidad que abarca desde efectos a nivel celular, como el daño mitocondrial y la apoptosis, hasta el desarrollo de síndrome metabólico, considerado por algunos autores como el intermediario entre cáncer y las enfermedades cardiovasculares ${ }^{50,51}$. Desde una perspectiva clínica, se puede predecir daño cardiaco inducido por anticancerígenos considerando la edad en la cual se recibe el tratamiento, la existencia de factores de riesgo cardiovascular y el uso de dosis acumulativas de éstos fármacos ${ }^{49}$. Los efectos negativos involucran tanto episodios de isquemia, como el desarrollo de arritmias que pueden afectar la función ventricular izquierda y terminar en el desarrollo de insuficiencia cardiaca ${ }^{47}$. La disfunción cardiaca relacionada con la terapia del cáncer ha sido definida como la disminución de la fracción de eyección del ventrículo izquierdo (FEVI) entre un 10 y 53\%52. Sin embargo, actualmente no existe un criterio único para definir la cardiotoxicidad mediada por antracilinas, toda vez que existe una incidencia del $12 \%$ de arritmias en pacientes tratados con éstos fármacos, mientras que el 2 a $4 \%$ presentan insuficiencia cardiaca y 9 a $11 \%$ presentan una caida en la fracción de eyección del ventrículo izquierdo ${ }^{49}$.

Considerando la sólida y abundante evidencia asociada al efecto cardiotóxico de fármacos anticancerígenos, el campo de investigación de la cardio-oncología enfoca actualmente sus esfuerzos en desarrollar estrategias que permitan proteger el corazón de la quimioterapia, más existe una necesidad imperativa de desarrollar terapias con aplicabilidad clínica efectiva. Los tratamientos con inhibidores de la enzima convertidora de angiotensina (IECAs), antagonistas beta-adrenérgicos, antagonistas del receptor de angiotensina II (ARAII), o dexrazoxane no son considerados de rutina para contrarrestar la cardiotoxicidad inducida por quimioterápicos ${ }^{49}$. Actualmente, el precondicionamiento isquémico puede ser una estrategia novedosa para proteger al miocardio de los efectos tóxicos de la doxorubicina ${ }^{53}$, pero se requieren más estudios para explorar su potencial cardioprotector en modelos animales in vivo, con el fin de, eventualmente, evaluar su efectividad en pacientes.

Evidencias epidemiológicas de una relación nueva entre enfermedad cardiovascular y cáncer Si bien se conoce mucho respecto a cómo el cáncer puede generar enfermedades cardiovasculares ${ }^{54}$, existe escasa evidencia concreta sobre la posibilidad de que las enfermedades cardiovasculares puedan inducir y promover cáncer. Las primeras sugerencias sobre esa posible relación se obtuvieron a partir de estudios observacionales. En 2013, Hasin et al, observaron que pacientes con insuficiencia cardiaca presentaban un mayor riesgo $(68 \%)$ de ser diagnosticados con cáncer en comparación con un grupo control. Además, estos pacientes tuvieron un mayor riesgo de morir (56\%) en comparación con pacientes con insuficiencia cardiaca sin cáncer ${ }^{55}$. El riesgo de aparición de cáncer comenzó 2 años después del diagnóstico de la insuficiencia cardiaca, siendo este mayor a los 4 años. Aunque estos hallazgos mostraban un diagnóstico más prematuro de cáncer en pacientes con insuficiencia cardiaca, vale recordar que la progresión del cáncer es un proceso relativamente lento. Por lo tanto, estos resultados se observaron con cautela porque se consideraba poco probable que la insuficiencia cardiaca por sí sola fuera la causa de la aparición temprana del cáncer. A pesar de estos antecedentes, los hallazgos de Hasin et al (2013) ${ }^{55}$ se reprodujeron con estudios prospectivos de cohortes de pacientes con insuficiencia cardiaca $^{56,57}$.

\section{Nuevas evidencias de una relación entre enfermedad cardiovascular y cáncer.}

El estudio de Meijers et al (2018) ${ }^{58}$ en la revista Circulation presentó las que, hasta el momento, son las evidencias más sólidas que asocian la insuficiencia cardiaca con la incidencia de cáncer, particularmente, cáncer de colón. Los autores obtuvieron esta información a partir de ratones APCmin, cuya modificación genética induce un aumento en la formación de adenomas intestinales de forma espontánea ${ }^{59,60}$. Estos ratones se sometieron a un procedimiento quirúrgico para inducir infarto agudo del miocardio (IAM) y seis semanas después, desarrollaron insuficiencia cardiaca que, como primer resultado interesante, estuvo acompañada por el incremento en el número y tamaño de los pólipos intestinales en comparación con el grupo control (ratones pseudo-operados o Sham). Además, los autores encontraron una asociación entre el crecimiento tumoral y eventos asociados con 
el remodelado cardiaco adverso desencadenados por la insuficiencia cardiaca, reflejado por el deterioro de la FEVI (FEVI $\approx 32 \%$ ) y fibrosis cardiaca.

Con el fin de descartar que la posible asociación fuera producto de las alteraciones hemodinámicas generadas por el corazón insuficiente, los autores transplantaron corazones procedentes tanto de ratones Sham, como de ratones APCmin sometidos a IAM una semana antes, en otros ratones APCmin recipientes no sometidos a infarto, mediante operación heterotópica en la región cervical. Los ratones APCmin que fueron transplantados con corazones infartados manifestaron un incremento en el número y tamaño de los pólipos en comparación con ratones que recibieron corazones procedentes de ratones Sham. Debido a que este hallazgo sugiere que el crecimiento tumoral podría deberse a factores secretados por el corazón insuficiente, los autores procedieron a buscar proteínas candidatas que suelen ser liberadas por el corazón luego de IAM y de esta manera seleccionaron cinco: serpina1 (alfa-1-antitripsina), serpina3 (alfa-1-antiquimotripsina), fibronectina, ceruloplasmina y PON1 (paraoxonasa 1). Los ratones APCmin con insuficiencia cardiaca mostraron niveles elevados de mRNA de estas proteínas a nivel de ventrículo izquier- do y, específicamente, SerpinA1 y SerpinA3 promovieron la proliferación en la línea celular HT-29 (células de adenocarcinoma colorrectal humano).

Meijers et al también determinaron si sus resultados eran extrapolables al contexto clínico y, en efecto, encontraron que los niveles plasmáticos de las cinco proteínas candidatas estaban elevados en 101 pacientes con insuficiencia cardiaca en comparación con 180 sujetos sanos que participaron en el estudio PREVEND (Prevention of Renal and Vascular End-stage Disease) ${ }^{61,62}$.

\section{Implicancias y proyecciones clínicas}

El alto riesgo de desarrollo de insuficiencia cardiaca en pacientes con cáncer es un fenómeno ampliamente aceptado en la práctica clínica. Sin embargo, los hallazgos de Meijers et al (2018) ${ }^{58}$ muestran cómo la insuficiencia cardiaca puede incidir significativamente en el desarrollo tumoral, hallazgo que muestra una nueva dirección hasta ahora inexplorada en la interrelación que existe entre estas dos enfermedades (Figura 2). Este estudio abre la puerta a nuevas investigaciones sobre la influencia que puedan ejercer otras enfermedades cardiovasculares en el desarrollo de cáncer, así como el papel de la IC, no sólo en la generación, sino también

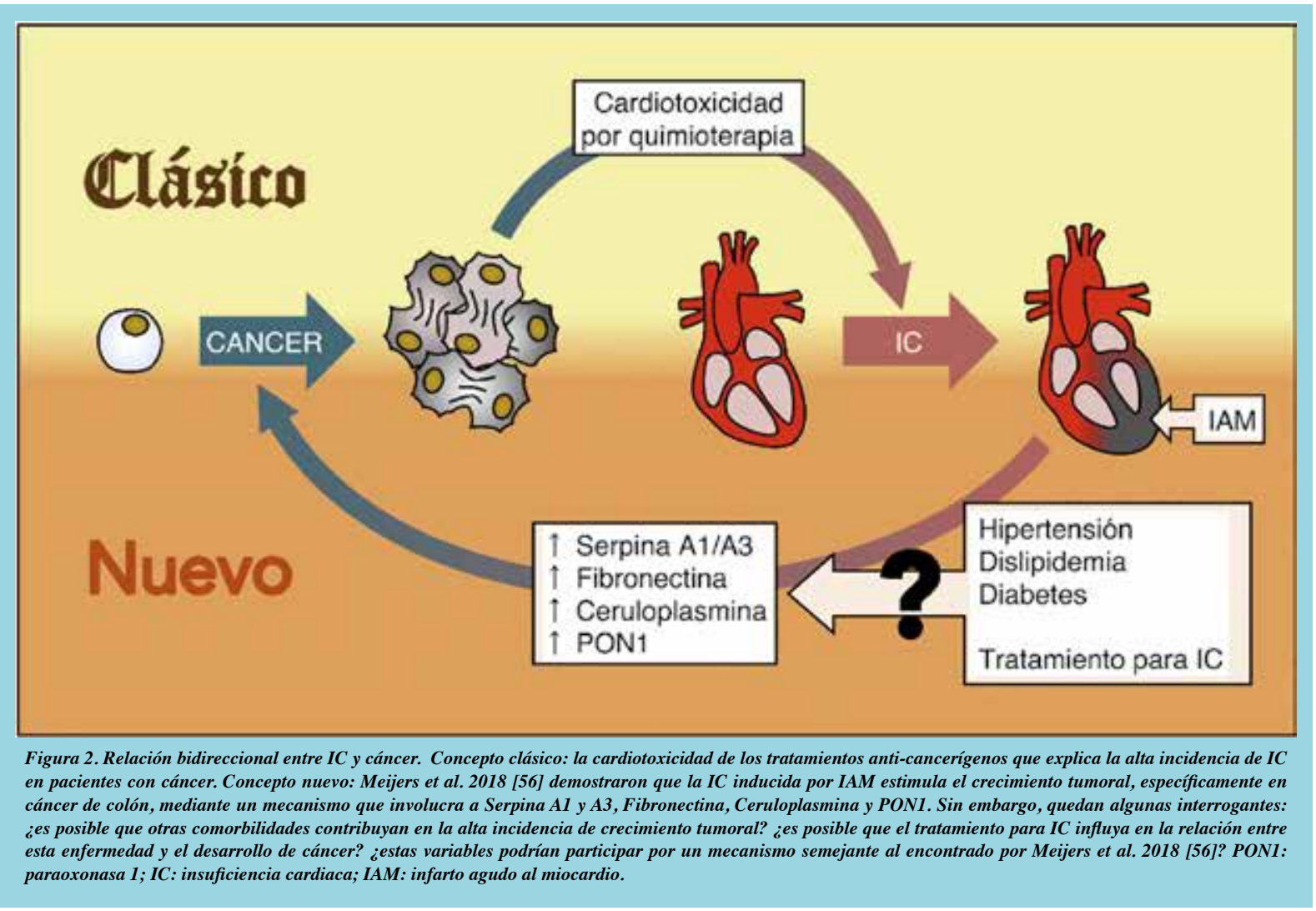


en el desarrollo metastásico en otros tipos de cáncer. Además, se requieren estudios clínicos que aclaren si la relación entre la IC y el desarrollo de cáncer es independiente de potenciales factores de confusión como las dislipidemias, la obesidad y el sedentarismo, entre otras, y que son más prevalentes en pacientes con $\mathrm{IC}^{63}$. Por otro lado, aunque Meijers et al ${ }^{58}$ presentaron cinco proteínas que explicarían el aumento del crecimiento tumoral en pacientes con IC, se requiere el estudio de los mecanismos subyacentes que involucran no solo a éstas, sino también a otras proteínas que podría estar secretando el corazón insuficiente y con potencial para uso como marcadores biológicos que faciliten el establecimiento de una escala de riesgo de cáncer en pacientes con IC.

En el contexto clínico, todavía quedan interrogantes que no fueron resueltas por Meijers et al, y estudios previos $55,56,58$ como es el caso de la influencia de los medicamentos empleados habitualmente en el tratamiento de la IC sobre el crecimiento tumoral, en particular, el efecto de los IECAs y bloqueadores del receptor de angiotensina, asociados con efectos carcinogénicos en varios estudios observacionales ${ }^{64}$. Además, es pertinente descartar la influencia de otras cardiopatías de base que acompañan o preceden a la IC así como el papel que desempeña el proceso inflamatorio crónico característico de esta enfermedad ${ }^{65}$.

Desde su aparición, la cardio-oncología se ha enfocado en la prevención, seguimiento y tratamiento de la IC en pacientes con cáncer. Ahora, tanto los estudios epidemiológicos de Hasin et al (2013 y 2016) 55,56 como los hallazgos recientes de Meijers et al $(2018)^{58}$, se convierten en los primeros pasos hacia lo que podría ser no sólo otro campo de investigación, sino también una nueva disciplina médica que tendría como tarea el seguimiento y tratamiento del cáncer en pacientes con IC.

\section{Agradecimientos}

Los autores agradecen a la Comisión Nacional de Investigación Científica y Tecnológica, CONICYT: Proyectos FONDAP 15130011 y FONDECYT 1161156 (S.L.), FONDECYT de Iniciación 11181000 (J.A.R.) y 3160226 (R.B.S.), PAI Subvención a Instalación en la Academia 201777170004 (R.B.S.), Beca Doctorado Nacional 63140060 (E.M.) y Beca Doctorado Exterior Colciencias-Colombia (E.M.).

\section{Referencias}

1 ALLEN LN, FEIGL AB. Reframing non-communicable diseases as socially transmitted conditions. Lancet Glob Health. 2017; 5: e644-e646. doi:10.1016/S2214-109X(17)30200-0.

2 ALLEN LN, FEIGL AB. What's in a name? A call to reframe non-communicable diseases. Lancet Glob Health. 2017; 5: e129-e130. doi:10.1016/S2213.

3 CAVALIN C, LESCOAT A. Reframing non-communicable diseases. Lancet Glob Health. 2017; 5: e1071. doi:10.1016/ S2214-109X(17)30327-3.
4 CAVALIN C, LESCOAT A, MACCHI O, REVEST M, ROSENTAL P-A, JÉGO P. Socioenvironmental factors of communicable and non-communicable diseases. Lancet Glob Health. 2017; 5: e487. doi:10.1016/S2214-109X(17)30150-X.

5 CHRISTAKIS NA, FOWLER JH. The Spread of Obesity in a Large Social Network over 32 Years. N Engl J Med. 2007; 357: 370-379.

6 ROTH GA, JOHNSON C, ABAJOBIR A, ABD-ALLAH F, ABERA SF, ABYU G, et al. Global, Regional, and National Burden of Cardiovascular Diseases for 10 Causes, 1990 
to 2015. J Am Coll Cardiol. 2017; 70: 1-25. doi:10.1016/j. jacc.2017.04.052.

7 MINISTERIO DE SALUD. Encuesta Nacional de Salud 20092010. https://www.minsal.cl/portal/url/item/bcb03d7bc28b64dfe040010165012d23.pdf. Consultado el 21 de enero de 2019

8 ENCUESTA NACIONAL DE SALUD 2016-2017 Primeros resultados. http://www.ipsuss.cl/ipsuss/site/artic/20171122/ asocfile/20171122142253/ens_2016_17_primeros_resultados. pdf. Cosultado el 16 de enero de 2019

9 OPS/OMS. OPS/OMS Chile - OMS publica una nueva edición del informe sobre el monitoreo de los progresos en enfermedades no transmisibles 2017. https://www.paho.org/chi/index. php?option $=$ com_content $\&$ view $=$ article $\&$ id $=953:$ oms - pu blica-una-nueva-edicion-del-informe-sobre-el-monitoreo-de-los-progresos-en-enfermedades-no-transmisibles\&Itemid=1005. Consultado el 9 de enero de 2019 .

10 INSTITUTO NACIONAL DE ESTADÍSTICAS. Anuario de Estadísticas vitales 2016. www.ine.cl/docs/default-source/demográficas-y-vitales/vitales/anuarios/2016/vitales-2016.pdf?sfvrsn=15. Consultado el 21 de enero de 2019.

11 MINISTERIO DE SALUD DE CHILE. Enfermedades No Transmisibles - Ministerio de Salud - Gobierno de Chile 2015. http://www.minsal.cl/enfermedades-no-transmisibles/. Consultado el 17 de julio de 2018 .

12 KOENE RJ, PRIZMENT AE, BLAES A, KONETY SH Shared Risk Factors in Cardiovascular Disease and Cancer. Circulation. 2016; 133: 1104-1114. doi: 10.1161/CIRCULATIONAHA.115.020406. n.d. doi:10.1161/CIRCULATIONAHA.115.020406.

13 MASOUDKABIR F, SARRAFZADEGAN N, GOTAY C, IGNASZEWSKI A, KRAHN AD, DAVIS MK, et al. Cardiovascular disease and cancer: Evidence for shared disease pathways and pharmacologic prevention. Atherosclerosis 2017; 263: 343-351. doi:10.1016/j.atherosclerosis.2017.06.001.

14 KORNILUK A, KOPER O, KEMONA H, DYMICKA-PIEKARSKA V. From inflammation to cancer. Ir J Med Sci. 2017; 186: 57-62. doi:10.1007/s11845-016-1464-0.

15 LOPEZ-CANDALES A, HERNÁNDEZ BURGOS PM, HERNANDEZ-SUAREZ DF, HARRIS D. Linking Chronic Inflammation with Cardiovascular Disease: From Normal Aging to the Metabolic Syndrome. J Nat Sci. 2017; 3.

16 RUPARELIA N, CHAI JT, FISHER EA, CHOUDHURY RP. Inflammatory processes in cardiovascular disease: a route to targeted therapies. Nat Rev Cardiol. 2017; 14: 133-144. doi:10.1038/nrcardio.2016.185

17 LEVY JMM, TOWERS CG, THORBURN A. Targeting autophagy in cancer. Nat Rev Cancer. 2017; 17: 528-542. doi:10.1038/nrc.2017.53

18 SANTANA-CODINA N, MANCIAS JD, KIMMELMAN AC. The Role of Autophagy in Cancer. Annu Rev Cancer Biol. 2017; 1: 19-39. doi:10.1146/annurev-cancerbio-041816-122338.

19 WHITE E. The role for autophagy in cancer. J Clin Invest. 2015; 125: 42-46. doi:10.1172/JCI73941.

20 SCHIATTARELLA GG, HILL JA. Therapeutic targeting of autophagy in cardiovascular disease. J Mol Cell Cardiol. 2016; 95: 86-93. doi:10.1016/j.yjmcc.2015.11.019.

21 LAVANDERO S, CHIONG M, ROTHERMEL BA, HILL JA. Autophagy in cardiovascular biology. J Clin Invest. 2015; 125: 55-64. doi:10.1172/JCI73943.

22 GARCÍA N, ZAZUETA C, AGUILERA-AGUIRRE L Oxidative Stress and Inflammation in Cardiovascular Disease. Oxid Med Cell Longev. 2017; 2017: 5853238. doi:10.1155/2017/5853238.

23 GILL JG, PISKOUNOVA E, MORRISON SJ. Cancer, oxidative stress, and metastasis. Cold Spring Harb Symp Quant Biol. 2016; 81:163-175. doi:10.1101/sqb.2016.81.030791.

24 SCHOTTENFELD D, BEEBE-DIMMER J. Chronic inflammation: a common and important factor in the pathogenesis of neoplasia. CA Cancer J Clin. 2006; 56: 69-83. doi:10.3322/ canjclin.56.2.69.

25 MULTHOFF G, MOLLS M, RADONS J. Chronic inflammation in cancer development. Front Immunol. 2012; 2: 1-17. doi:10.3389/fimmu.2011.00098.

26 GOLIA E, LIMONGELLI G, NATALE F, FIMIANI F, MADDALONI V, PARIGGIANO I, et al. Inflammation and cardiovascular disease: From pathogenesis to therapeutic target. Curr Atheroscler Rep. 2014; 16. doi:10.1007/s11883-014-0435-z.

27 WAHL SM, WONG H, MCCARTNEYDFRANCIS N. Role of growth factors in inflammation and repair. J Cell Biochem. 1989; 40: 193-199. doi:10.1002/jcb.240400208.

28 SAVIC-RADOJEVIC A, RADOVANOVIC S, PEKMEZOVIC T, PLJESA-ERCEGOVAC M, SIMIC D, DJUKIC T, et al. The Role of Serum VCAM- 1 and TNF- $\alpha$ as Predictors of Mortality and Morbidity in Patients with Chronic Heart Failure. J Clin Lab Anal. 2013; 27: 105-112. doi:10.1002/jcla.21570.

29 VERDEJO H, ROLDAN J, GARCIA L, DEL CAMPO A, BE- 
CERRA E, CHIONG M, et al. Systemic vascular cell adhesion molecule-1 predicts the occurrence of post-operative atrial fibrillation. Int J Cardiol. 2011; 150: 270-276. doi:10.1016/j. ijcard.2010.04.033.

30 YAMADA Y, ARAO T, MATSUMOTO K, GUPTA V, TAN W, FEDYNYSHYN J, et al. Plasma concentrations of VCAM-1 and PAI-1: A predictive biomarker for post-operative recurrence in colorectal cancer. Cancer Sci. 2010; 101: 1886-1890. doi:10.1111/j.1349-7006.2010.01595.x.

31 TERPOS E, MIGKOU M, CHRISTOULAS D, GAVRIATOPOULOU M, ELEUTHERAKIS-PAPAIAKOVOU E, KANELLIAS N, et al. Increased circulating VCAM-1 correlates with advanced disease and poor survival in patients with multiple myeloma: reduction by post-bortezomib and lenalidomide treatment. Blood Cancer J. 2016; 6: e428. doi:10.1038/ bcj.2016.37.

32 FAROOQI AA, SIDDIK ZH. Platelet-derived growth factor (PDGF) signalling in cancer: rapidly emerging signalling landscape. Cell Biochem Funct 2015;33:257-65. doi:10.1002/ cbf. 3120 .

33 DOMOUZOGLOU EM, NAKA KK, VLAHOS AP, PAPAFAKLIS MI, MICHALIS LK, TSATSOULIS A, et al. Fibroblast growth factors in cardiovascular disease: The emerging role of FGF21. Am J Physiol - Hear Circ Physiol. 2015; 309: H1029-H1038. doi:10.1152/ajpheart.00527.2015.

34 KORC M, FRIESEL RE. The role of fibroblast growth factors in tumor growth. Curr Cancer Drug Targets. 2009; 9: 639-651. doi:10.2174/156800909789057006.

35 MASSAGUÉ J. TGF $\beta$ in cancer. Cell. 2008; 134: 215-230. doi:10.1016/j.cell.2008.07.001.

36 PARDALI E, TEN DIJKE P. TGF $\beta$ signaling and cardiovascular diseases. Int J Biol Sci 2012; 8: 195-213. doi:10.7150/ ijbs.3805.

37 DING Y BIN, CHEN GY, XIA JG, ZANG XW, YANG HY, YANG L. Association of VCAM-1 overexpression with oncogenesis, tumor angiogenesis and metastasis of gastric carcinoma. World J Gastroenterol. 2003; 9: 1409-1414. doi:10.3748/ wjg.v9.i7.1409.

38 KONG DH, KIM YK, KIM MR, JANG JH, LEE S. Emerging roles of vascular cell adhesion molecule-1 (VCAM-1) in immunological disorders and cancer. Int J Mol Sci. 2018; 19: 13-17. doi:10.3390/ijms19041057.

39 LEY K, HUO Y. VCAM-1 is critical in atherosclerosis. J Clin Invest 2001; 107: 1209-1210. doi:10.1172/JCI13005.
40 PARK JG, RYU SY, JUNG IH, LEE YH, KANG KJ, LEE MR, et al. Evaluation of VCAM-1 antibodies as therapeutic agent for atherosclerosis in apolipoprotein E-deficient mice. Atherosclerosis. 2013; 226: 356-363. doi:10.1016/j.atherosclerosis.2012.11.029.

41 REUTER S, GUPTA SC, CHATURVEDI MM, AGGARWAL BB. Oxidative stress, inflammation, and cancer: How are they linked? Free Radic Biol Med. 2011; 49: 1603-1616. doi:10.1016/j.freeradbiomed.2010.09.006.

42 ZHANG N, ANDRESEN BT, ZHANG C. Inflammation and reactive oxygen species in cardiovascular disease. World J Cardiol. 2010; 2: 408-410. doi:10.4330/wjc.v2.i12.408.

43 PANTH N, PAUDEL KR, PARAJULI K. Reactive Oxygen Species: A Key Hallmark of Cardiovascular Disease. Adv Med. 2016; 2016: 9152732. doi:10.1155/2016/9152732.

44 GLICK D, BARTH S, MACLEOD KF. Autophagy : cellular and molecular mechanisms. J Pathol. 2010; 221: 3-12. doi:10.1002/path.2697.Autophagy.

45 YONEKAWA T, THORBURN A. Autophagy and cell death. Essays Biochem. 2013; 55: 105-117. doi:10.1042/bse0550105.

46 HARRIS J.Autophagy and cytokines. Cytokine. 2011; 56: 140144. doi:10.1016/j.cyto.2011.08.022.

47 HAMO CE, BLOOM MW. Cancer and Heart Failure: Understanding the Intersection. Cit Card Fail Rev. 2017; 23: 66-70. doi:10.15420/cfr.2016:24:2.

48 ALEMAN BMP, MOSER EC, NUVER J, SUTER TM, MARALDO M V, SPECHT L, et al. Cardiovascular disease after cancer therapy. Eur J Cancer Suppl. 2014; 12: 18-28. doi:10.1016/J.EJCSUP.2014.03.002.

49 MCGOWAN J V, CHUNG R, MAULIK A, PIOTROWSKA I, WALKER JM, YELLON DM. Anthracycline Chemotherapy and Cardiotoxicity. Cardiovasc Drugs Ther. 2017; 31: 63-75. doi:10.1007/s10557-016-6711-0.

50 FLORESCU M, CINTEZA M, VINEREANU D. Chemotherapy-induced Cardiotoxicity. Maedica (Buchar). 2013; 8: 59-67.

51 WESTERINK NL, NUVER J, LEFRANDT JD, VRIELING AH, GIETEMA JA, WALENKAMP AME. Cancer treatment induced metabolic syndrome: Improving outcome with lifestyle. Crit Rev Oncol / Hematol. 2016; 108: 128-136. doi:10.1016/j.critrevonc.2016.10.011.

52 PLANA JC, GALDERISI M, BARAC A, EWER MS, KY B, SCHERRER-CROSBIE M, et al. Expert Consensus for Multimodality Imaging Evaluation of Adult Patients during and 
after Cancer Therapy: A Report from the American Society of Echocardiography and the European Association of Cardiovascular Imaging. J Am Soc Echocardiogr. 2014; 27: 911-939. doi:10.1016/j.echo.2014.07.012.

53 MAULIK A, DAVIDSON SM, PIOTROWSKA I, WALKER M, YELLON DM. Ischaemic Preconditioning Protects Cardiomyocytes from Anthracycline-Induced Toxicity via the PI3K Pathway. Cardiovasc Drugs Ther. 2018; 32: 245-253. doi:10.1007/s10557-018-6793-y.

54 YEH ETH, CHANG H-M. Oncocardiology-Past, Present, and Future: A Review. JAMA Cardiol. 2016; 1: 1066-1072. doi:10.1001/jamacardio.2016.2132.

55 HASIN T, GERBER Y, MCNALLAN SM, WESTON SA, KUSHWAHA SS, NELSON TJ, et al. Patients With Heart Failure Have an Increased Risk of Incident Cancer. J Am Coll Cardiol. 2013; 62: 881-886. doi:10.1016/j.jacc.2013.04.088.

56 HASIN T, GERBER Y, WESTON SA, JIANG R, KILLIAN JM, MANEMANN SM, et al. Heart Failure After Myocardial Infarction Is Associated With Increased Risk of Cancer. J Am Coll Cardiol. 2016; 68: 265-271. doi:10.1016/j. jacc.2016.04.053.

57 BANKE A, SCHOU M, VIDEBAEK L, MØLLER JE, TORP-PEDERSEN C, GUSTAFSSON F, et al. Incidence of cancer in patients with chronic heart failure: a long-term follow-up study. Eur J Heart Fail. 2016; 18: 260-266. doi:10.1002/ ejhf.472.

58 MEIJERS WC, MAGLIONE M, BAKKER SJL, OBERHUBER R, KIENEKER LM, DE JONG S, et al. The Failing Heart Stimulates Tumor Growth by Circulating Factors. Circulation. 2018; 138: 678-691. doi: 10.1161/CIRCULATIONAHA.117.030816.
59 MOSER AR, LUONGO C, GOULD KA, MCNELEY MK, SHOEMAKER AR, DOVE WF. ApcMin: a mouse model for intestinal and mammary tumorigenesis. Eur J Cancer. 1995; 31A: 1061-1064.

60 HANKEY W, FRANKEL WL, GRODEN J. Functions of the APC tumor suppressor protein dependent and independent of canonical WNT signaling: implications for therapeutic targeting. Cancer Metastasis Rev. 2018; 37:159-172. doi:10.1007/ s10555-017-9725-6.

61 SCHROTEN NF, RUIFROK WPT, KLEIJN L, DOKTER MM, SILLJÉ HH, LAMBERS HEERSPINK HJ, et al. Shortterm vitamin D3 supplementation lowers plasma renin activity in patients with stable chronic heart failure: an open-label, blinded end point, randomized prospective trial (VitD-CHF trial). Am Heart J. 2013; 166: 357-364. doi:10.1016/j. ahj.2013.05.009.

62 HILLEGE HL, JANSSEN WM, BAK AA, DIERCKS GF, GROBBEE DE, CRIJNS HJ, et al. Microalbuminuria is common, also in a nondiabetic, nonhypertensive population, and an independent indicator of cardiovascular risk factors and cardiovascular morbidity. J Intern Med. 2001; 249: 519-526.

63 GREENLAND P. Major Risk Factors as Antecedents of Fatal and Nonfatal Coronary Heart Disease Events. JAMA. 2003; 290: 891. doi:10.1001/jama.290.7.891.

64 SINGH A, BANGALORE S. Which, if any, antihypertensive agents cause cancer? Curr Opin Cardiol. 2012; 27: 374-380. doi:10.1097/HCO.0b013e328353bc4f.

65 LAUER MS. Elements of Danger - The Case of Medical Imaging. N Engl J Med. 2009; 361: 841-843. doi:10.1056/NEJMp0904735. 